\title{
Changes in platelet aggregation after suspension of aspirin therapy
}

\author{
Kojiro Furukawa, MD, and Hitoshi Ohteki, MD, Saga, Japan
}

A spirin medication is often suspended from 5 to 7 days before cardiac surgery because of the risk of increased perioperative blood loss. ${ }^{1}$ However, Dacey and colleague ${ }^{2}$ recently reported that preoperative aspirin use among patients undergoing coronary artery bypass grafting appears to be associated with a decreased risk of mortality, without any significant increases in hemorrhage, blood product requirements, or related morbidities. Whether aspirin use or nonuse is the more efficacious strategy remains a controversial question. Furthermore, from the viewpoint of hard data, there are no clinical reports about changes in platelet aggregation after stopping aspirin.

\section{Patients and Methods}

To standardize the platelet aggregation test, we used the grading curve method. ${ }^{3}$ Four concentrations $(0.25,0.5,1.0$, and $2.0 \mu \mathrm{g} /$ $\mathrm{mL}$ ) of collagen (an aggregation inducer) were plotted along the horizontal axis, and the corresponding percentages of aggregation ( 5 minutes after collagen administration) were plotted along the vertical axis. Rates of aggregation were measured with light transmission by the method of Borns and Cross. ${ }^{4}$ We used the platelet aggregator threshold index (PATI), which is obtained from the grading curve, to represent the degree of platelet aggregation. The PATI is the concentration of collagen that corresponds to $50 \%$ of maximum aggregation $^{3}$ (Figure 1). It correlates well with the minimum concentration of irreversible aggregation, as the platelet aggregation inducer. ${ }^{3}$ The grading curve and PATI were measured automatically with a PAM 8T aggregometer (Mebanics Inc, Tokyo, Japan). Twenty-two consecutively scheduled cardiac surgical patients, each of whom received $81 \mathrm{mg}$ of aspirin, were included (13 male and 9 female patients, mean age $65 \pm 9.3$ years, range 47-77 years). Fifteen were undergoing coronary artery bypass grafting, and the remaining 7 were undergoing valve surgery. PATIs were measured before and at 1, 2, 3, and 5 days after stopping aspirin. All values were expressed as mean $\pm \mathrm{SD}$. The unpaired $t$ test was performed for comparisons between the groups.

\section{Results}

The PATIs (in $\mu \mathrm{g} / \mathrm{mL}$ ) before and $1,2,3$, and 5 days after stopping aspirin were $3.6 \pm 2.0,1.7 \pm 1.2,0.9 \pm 0.8$, and $0.6 \pm 0.5$,

\footnotetext{
From the Department of Cardiovascular Surgery, Saga Prefectural Hospital, Koseikan, Saga, Japan.

Received for publication Dec 16, 2003; accepted for publication Dec 30, 2003.

Address for reprints: Kojiro Furukawa, MD, Department of Thoracic and Cardiovascular Surgery, Faculty of Medicine, Saga University, 5-1-1 Nabeshima, Saga City 840-8571, Japan (E-mail: furukawa@bcm.tmc.edu).

J Thorac Cardiovasc Surg 2004;127:1814-5

$0022-5223 / \$ 30.00$

Copyright @ 2004 by The American Association for Thoracic Surgery

doi:10.1016/j.jtcvs.2003.12.025
}

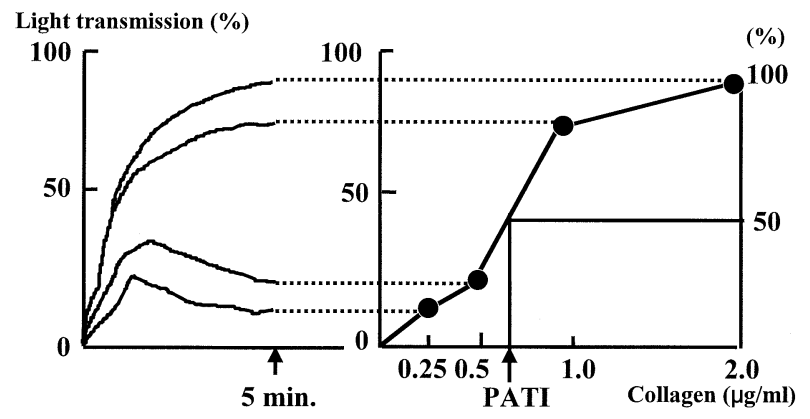

Figure 1. Aggregation curve (left) and grading curve and definition of PATI (right). See text for details.

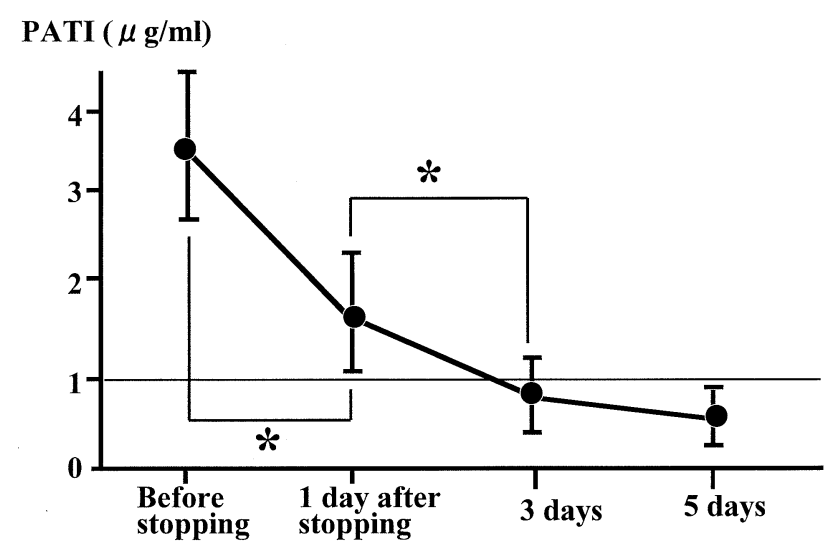

Figure 2. Overall results. (Asterisk indicates $P<.05$.)

respectively. PATIs decreased significantly from before to 1 day after stopping aspirin $(P=.0007)$ and from 1 day to 3 days after stopping $(P=.01)$ (Figure 2).

\section{Discussion}

There are many evaluation methods for platelet aggregation, but none of these has been defined as the standard. The evaluation of platelet aggregation by a grading curve has been developed and used in Japan. This method seems to enable easy determination of the degree of aggregation and is considered to be useful for monitoring platelet aggregation during the administration of antiplatelet drugs. ${ }^{3}$ The PATI, which can be read from the grading curve, correlates well with minimum concentrations of irreversible aggregation. ${ }^{3}$ Therefore a low PATI indicates accelerated aggregation, whereas a high one indicates reduced aggregation.

Aspirin inhibits the production of thromboxane $\mathrm{A}_{2}$ by irreversibly acetylating cyclooxygenase in the platelets. Thromboxane $\mathrm{A}_{2}$ is essential to the normal clotting process initiated by the platelets. 
The loss of cyclooxygenase in platelets, which lack the capacity for new protein synthesis, constitutes a loss of function for the remainder of their lifetime. The average life span of platelets is 7 days; therefore the conventional practice is to stop aspirin therapy 1 week before the planned procedure. However, the results of this study show that inhibition of platelet aggregation with aspirin vanishes earlier than expected. The life span of the platelet and platelet aggregation as a whole need to be understood separately. Although it may take 10 days for the total platelet population to be renewed and thus restore normal cyclooxygenase activity, it has been shown in vitro that if as few as $20 \%$ of total platelets have normal cyclooxygenase activity, aggregation would remain normal. ${ }^{5}$ Our study has confirmed this finding in the clinical situation.

This study has several limitations. One of these is the small study population. We therefore should extend this study and investigate the outcomes. Another limitation concerns aspirin dosages, which were low $(81 \mathrm{mg} / \mathrm{d})$ in this study. If the aspirin dosage had been higher, the speed of recovery of aggregation might have been different.

In conclusion, this study indicates that inhibition of platelet aggregation with an aspirin fell quickly at 1 day after discontinu- ation and vanished after 3 days. Therefore, in the presence of preoperative aspirin therapy, stopping at 3 days before surgery might be optimal for preventing bleeding complications. In addition, this study result should be considered when comparing aspirin users versus nonusers in future investigations into the effects of aspirin in cardiac surgery.

\section{References}

1. Sethi GK, Copeland JG, Goldman S, Moritz T, Zandina K, Henderson WG, et al. Implications of preoperative administration of aspirin in patients undergoing coronary artery bypass grafting. J Am Coll Cardiol. 1990; 15:15-20.

2. Dacey LJ, Munoz JJ, Johnson ER, Leavitt BJ, Maloney CT, Morton JR, et al. Effect of preoperative aspirin use on mortality in coronary artery bypass grafting patients. Ann Thorac Surg. 2000;70:1986-90.

3. Mase K, Yanagihara F, Asada T, Yamanaka Y, Yoshioka M, Yasunaga K. Evaluation of platelet aggregation test by grading curve. Jpn J Clin Pathol. 1992;40:857-62.

4. Borns CV, Cross MJ. The aggregation of blood platelets. J Physiol. 1963;168:178-95.

5. Bradlow BA, Chetty N. Dosage frequency for suppression of platelet function by low dose aspirin therapy. Thromb Res. 1982;27:99-110.

\title{
Surgical pitfalls during explantation of a thoracic aortic endoprosthesis
}

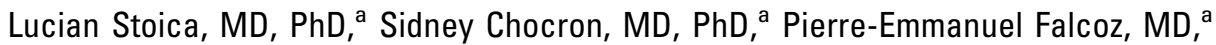 \\ Jean-François Bonneville, MD, ${ }^{b}$ and Joseph-Philippe Etievent, MD, ${ }^{a}$ Besançon, France
}

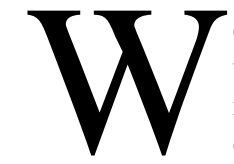

e report our experience with explantation of a thoracic aortic endoprosthesis 2 years after the initial procedure. This graft was explanted because of failure of endovascular treatment of an aneurysm that developed after surgical treatment of a type A aortic dissection. Crossclamping of the aortic arch was impossible, and surgery had to be performed with the patient under circulatory arrest. The procedures performed are time-consuming under circulatory arrest, making distal aortic arch surgery more complex after endovascular repair than when performed at primary surgery.

\footnotetext{
From the Departments of Thoracic and Cardio-Vascular Surgery ${ }^{\mathrm{a}}$ and

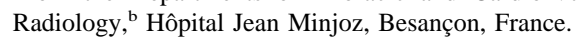

Received for publication Dec 13, 2003; accepted for publication Dec 30, 2003.

Address for reprints: Lucian Stoica, MD, PhD, Department of Thoracic and Cardiovascular Surgery, Hôpital Jean Minjoz, 3 Blvd Fleming, Besançon, France 25000 (E-mail: 1.stoica@ voila.fr).

J Thorac Cardiovasc Surg 2004;127:1815-7

$0022-5223 / \$ 30.00$

Copyright $\odot 2004$ by The American Association for Thoracic Surgery

doi:10.1016/j.jtcvs.2003.12.026
}

\section{Clinical Summary}

A 48-year-old man was operated on for acute type-A aortic dissection. We performed a supracoronary graft replacement of the ascending aorta under hypothermic circulatory arrest with cerebral retroperfusion through the superior vena cava. ${ }^{1}$ We found only one intimal tear on the ascending aorta. The aortic valve was spared. One year later, magnetic resonance imaging control revealed a perfused false lumen with dilatation of the aorta to 53 $\mathrm{mm}$. Aortography showed a communication between the two lumens just below the origin of the left subclavian artery. A Talent endoluminal stent graft (World Medical Corp, Sunrise, Fla) was deployed 17 months after the first operation. It covered the origin of the left subclavian artery, and the uncovered part was placed facing the origin of the left common carotid artery. Because the false lumen was still being perfused by the left subclavian artery, the origin of the artery was completely obstructed with coils (Figure 1). The treatment was completed with a bypass between the left carotid artery and the left subclavian artery to avoid left arm ischemia.

Two years after endovascular treatment, the false lumen was still perfused and the diameter of the postisthmic thoracic aorta had increased to $57 \mathrm{~mm}$ (Figure 1). We performed the explantation of the endoprosthesis through a left thoracotomy with the patient 